\title{
Wire bond vibration of Forward Pixel Tracking Detector of CMS
}

\author{
M. Atac ${ }^{\text {*a }}$, B. Gobbi ${ }^{\text {b }}$, S. Kwan ${ }^{\text {a }}$, Y. Pischalnikov ${ }^{\text {a }}$, E. Spencer ${ }^{\text {b }}$, \\ G. Sellberg ${ }^{a}$, V. Pavlicek ${ }^{a}$ \\ ${ }^{\text {a }}$ Fermi National Accelerator Lab ${ }^{\dagger}$, Batavia, IL, 60510, USA. \\ ${ }^{\mathbf{b}}$ Northwestern University, Evanston, IL, 60208, USA \\ email:matac@fnal.gov
}

\begin{abstract}
Wire bonds of the Forward Pixel (FPix) tracking detectors are oriented in the direction that maximizes Lorentz Forces relative to the 4 Tesla field of the Compact Muon Solenoid (CMS) Detector's magnet. The CMS Experiment is under construction at the Large Hadron Collider at CERN, Geneva, Switzerland. We were concerned about Lorentz Force oscillating the wires at their fundamental frequencies and possibly fracturing or breaking them at their heels, as happened with the CDF wire bonds. This paper reports a study to understand what conditions break such bonds.
\end{abstract}

Keywords: Particle Tracking Detectors, Wire bond vibrations, Lorentz Force

\footnotetext{
* Corresponding Author

${ }^{\dagger}$ Operated by Universities Research Association Inc., under Contract No. DE-AC02-76CH3000 with the United States Department of Energy.
} 


\section{Introduction}

The CMS Experiment [Ref.1] is under construction at the Large Hadron Collider at CERN, Geneva, Switzerland. Figure 1 shows the overall structure of the detector [Ref.2], while the pixel tracker layout is shown in Fig.2. The magnetic field of the CMS magnet is going to be perpendicular to the planes of many of the wire bond wires of the FPix discs [Ref. 3].

We were concerned about Lorentz Force oscillating the wires at their fundamental frequencies and possibly fracturing or breaking them at their heels, as happened with the CDF wire bonds [Ref.4]. Figure 3 shows the wire bonds between the Readout Chip (ROC), the very high density interconnect (VHDI), and high density interconnect (HDI). We expect maximum Lorentz Force acting on these wires during the $\mathrm{AC}$ part of the currents going through them during the readout cycles.

Tests were carried out at Fermilab to understand the conditions that could break the wire bonds by Lorentz Force induced motion in the wire bond wires. Typical failures are fracturing at one of the heels of the wire bond. Motion is enhanced at the fundamental mechanical resonance frequency. The tests were done in a conventional $2 \mathrm{~T}$ field calibration magnet at the Magnet Test Facility (MTF), as shown in Fig. 4.

\section{Tests results}

Initially, we used wire bonded readout chips in charge injection mode, sweeping the pulse rate between $100 \mathrm{~Hz}$ and 20 $\mathrm{kHz}$ for half a day slowly varying the pulse rate. We could not observe vibrations. In this case the AC part of the currents was about $35 \mathrm{~mA}$ amplitude and $56 \mathrm{~ns}$ in full width. Lack of vibration convinced us that the energy going through the wire 
bonds was not sufficient to oscillate the wires by the Lorentz Force. The maximum field which could be reached with the magnet was 2.2 Tesla due to the limit to operate the microscope with the CCD camera attached in the gap as shown in the figure. We then decided to use specially designed boards having aluminum wire bond wires of 25 micron thickness and $3 \mathrm{~mm}$ length, precisely with the height and length of the wire bond wires used for the readout chips (ROC) of the FPix (Fig. 4). This layout simplified the testing and observation but we could not move the microscope lens enough to close the gap and increase the field.

The electronic set up is shown in Fig. 5. Two signal generators were used for the tests, one for triggering the pulser and one for sweeping the frequencies and the second one provided the pulse currents through the wire bond wires. Input and output pulse shapes and the magnified image of the wire bond wire can be seen in the figure. A better picture of the magnified wire is shown in Fig. 6.

It was very easy to find the fundamental vibration frequency and break the wire bonds at the heels when we used CDF-like pulses $(2.3 \mu \mathrm{sec}$ wide and $200 \mathrm{~mA})$. Then we did a systematic study by using twice the expected operational current setting of $35 \mathrm{~mA}$ that is expected from the ROC chips to compensate for our test magnetic field being one-half of the 4 Tesla field of the CMS magnet, and varied the pulse width in steps, searching for the fundamental frequency of vibration. We kept the pulse widths constant for length of time to find out if the wire bond could break at one of the heels. We did this for several wire bonds and the following table summarizes one of the examples. 


\begin{tabular}{lcc} 
Time (hrs) & Pulse width (ns) & Fundamental frequency (kHz) \\
\hline $15: 15$ & 100 & 16.65 \\
$15: 55$ & 200 & 16.65 \\
$15: 56$ & 300 & 16.65 \\
$15: 59$ & 400 & 16.65 \\
$16: 04$ & 500 & 16.65 \\
$16: 07$ & 600 & 16.64 \\
$16: 12$ & 800 & 16.35 \\
$16: 15$ & $1 \mu \mathrm{s}$ & 16.28 \\
$16: 17$ & $1.2 \mu \mathrm{s}$ & 16.10 \\
$16: 22-26$ & $1.4 \mu \mathrm{s}$ & wire breaks off
\end{tabular}

It is evident that one of the heels started to fracture at the $\mathbf{6 0 0}$ ns pulse width and after few minutes of vibration it broke off as shown in Fig.7. The table clearly indicates that there is an energy threshold and that it was necessary to provide sufficient vibration to break our wire bonds by the Lorentz force at the heels in the 4 Tesla equivalent magnetic field condition. We saw similar results with all of the wire bond failures that were observed. The resonance frequencies were found to be sharply defined up to the energy at which fracturing started.

\section{Acknowledgement}

The authors would like to express their appreciation to Dr. M. Kubantsev for his contribution to the paper and also to the Magnet Test Facility personnel for their support during the tests. 


\section{References}

1. CMS Tracker Project Technical Design Report, CERN/LHCC 98-6.

2. CMSIM, http://cmsdoc.cern.ch/cms/outreach/html/CMSdocum ents/DetectorDrawings/fromGEANT/cms_complete_l abelled.gif

3. US-CMS Review, J. Butler, 2/2006, http://indico.cern.ch/conferencesDisplay.py?confId=a 06771

4. G. Bolla et al., Nucl. Instr. and Meth. A 518(2004) 277-280 
Figure 1. Drawing of the CMS Detector at the Large Hadron Collider at CERN

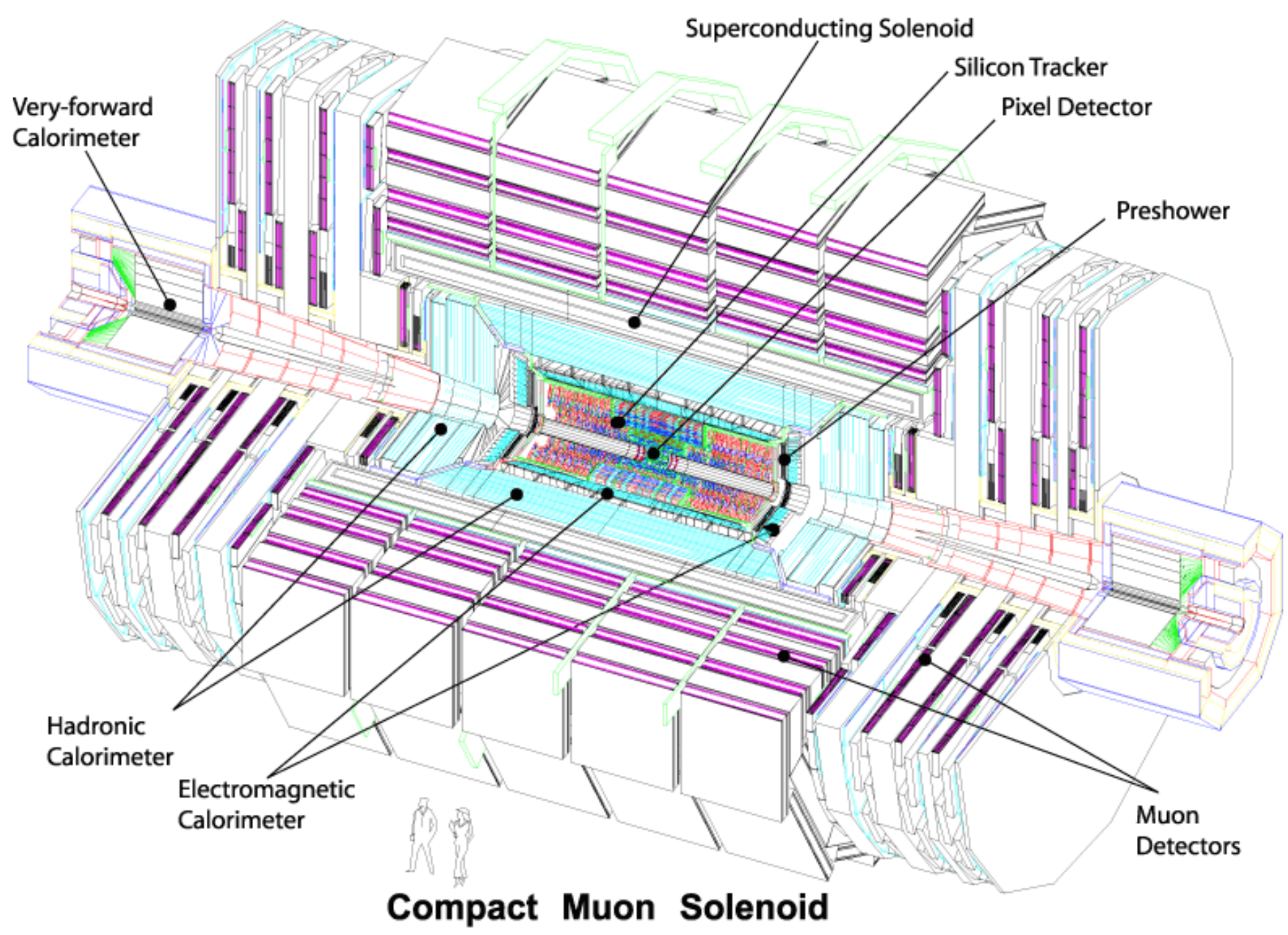




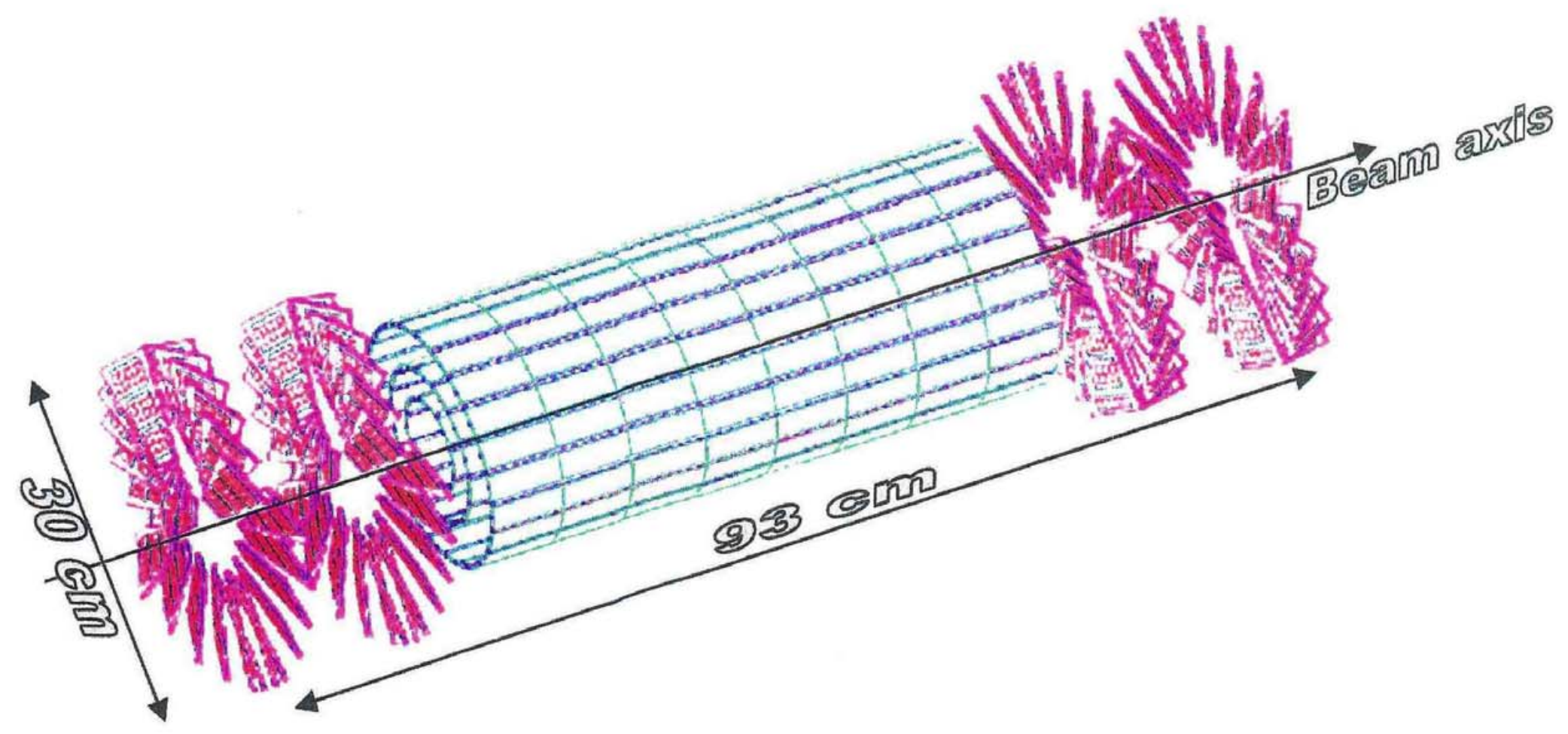

Figure 2. Simulation drawing of the Pixel Tracker Detector. 


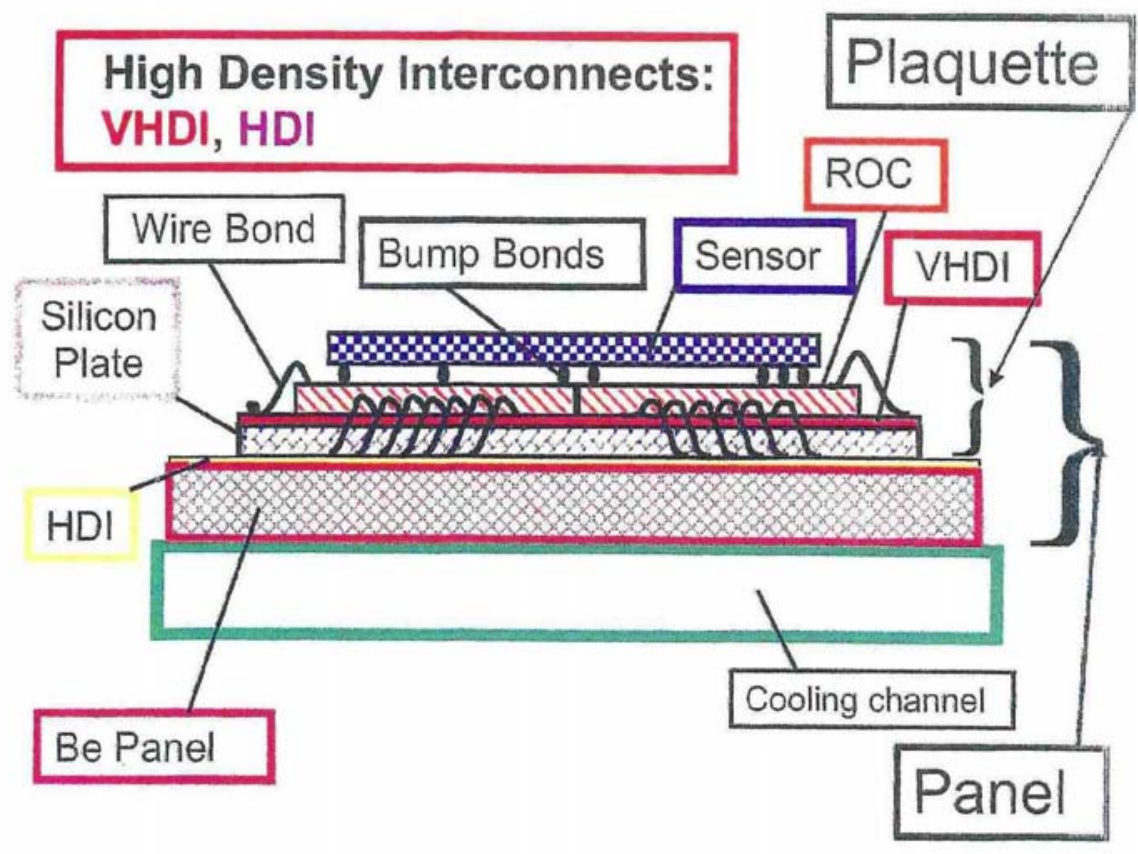

Figure 3. Wire bonds between the Readout Chip (ROC) and the very high density interconnect (VHDI), and the VHDI and high density interconnect (HDI). 


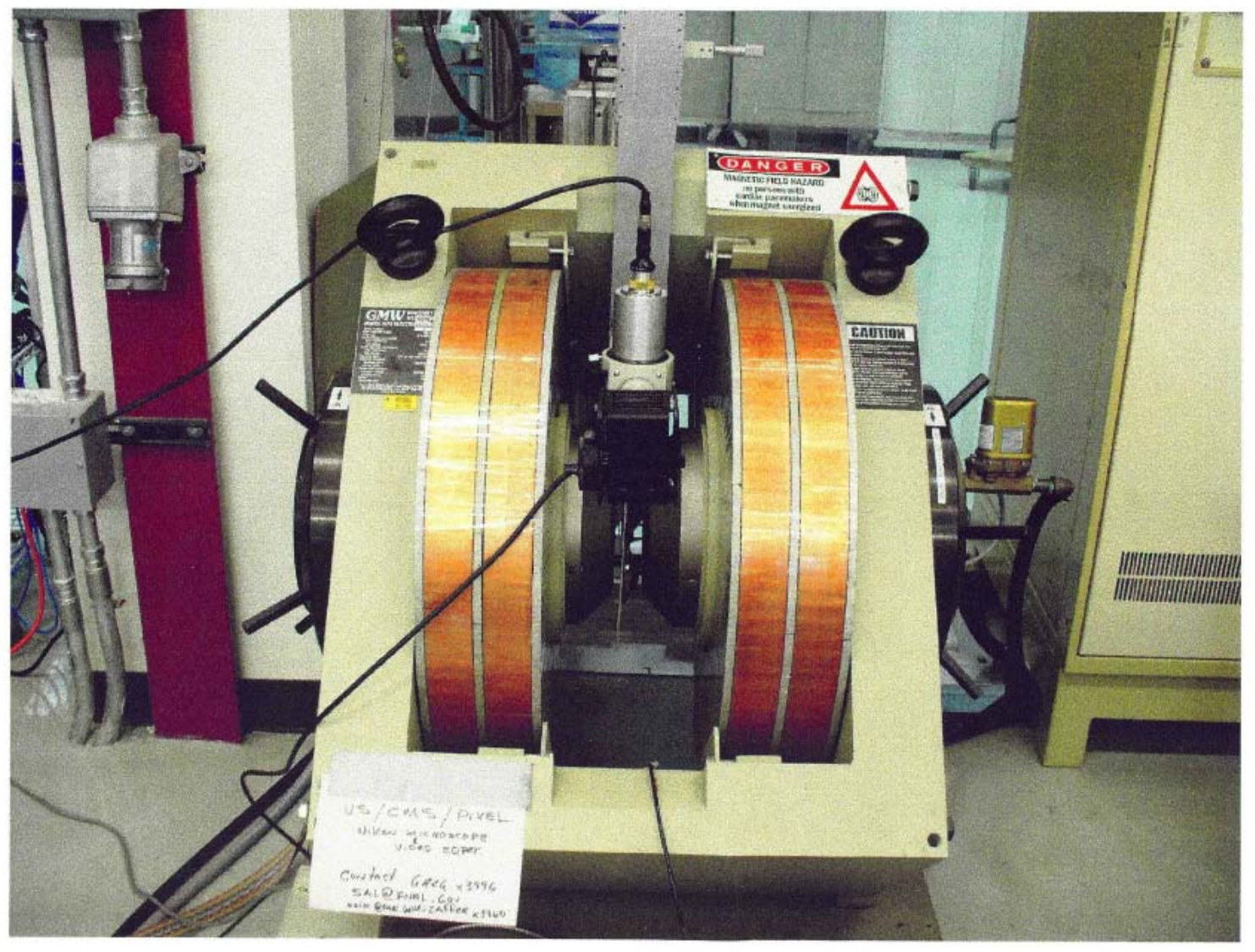

Figure 4. A picture of the calibration magnet at the Magnet Test Facility (MTF) at Fermilab. 


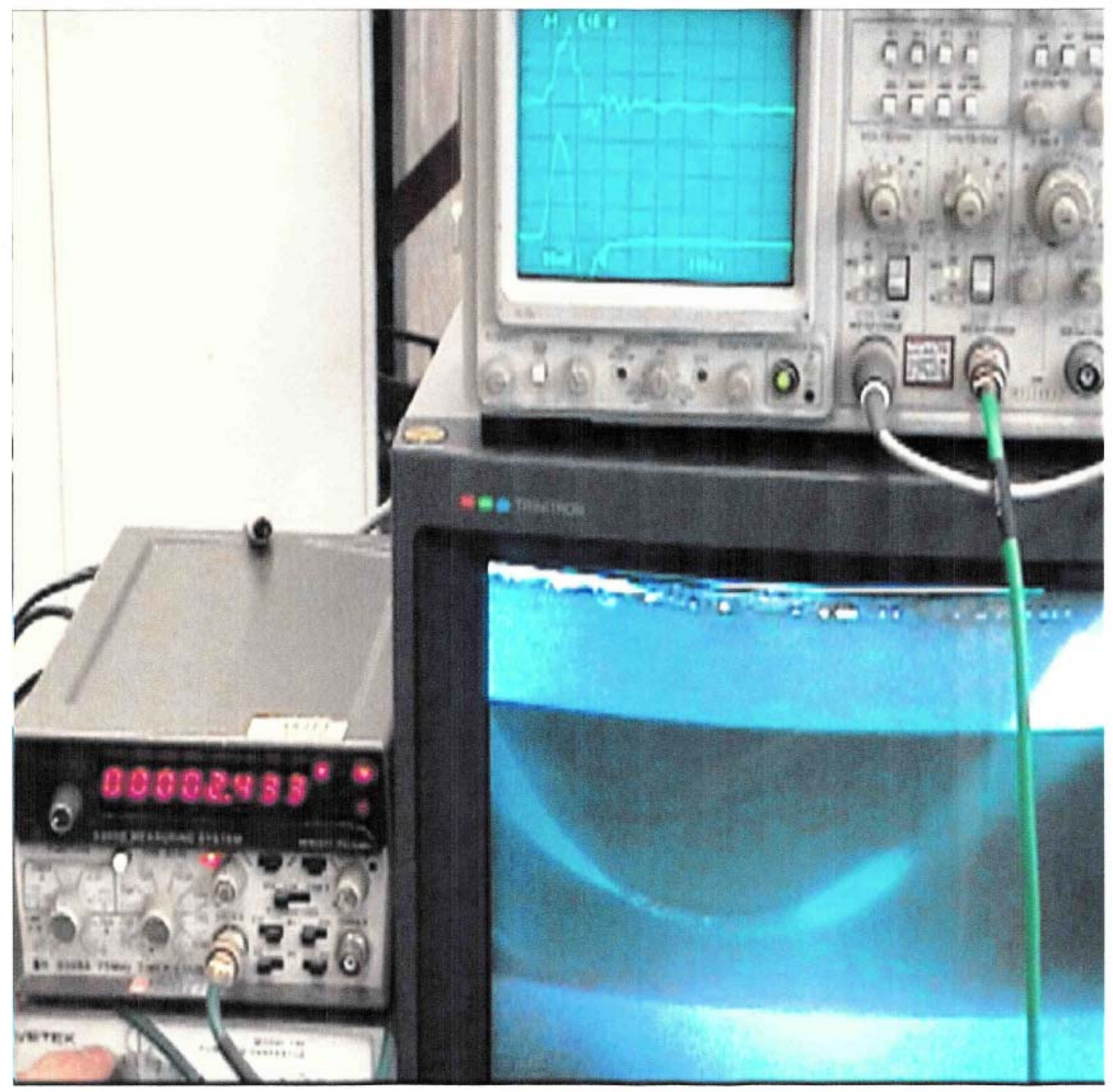

Figure 5 A picture of the electronic set up. 


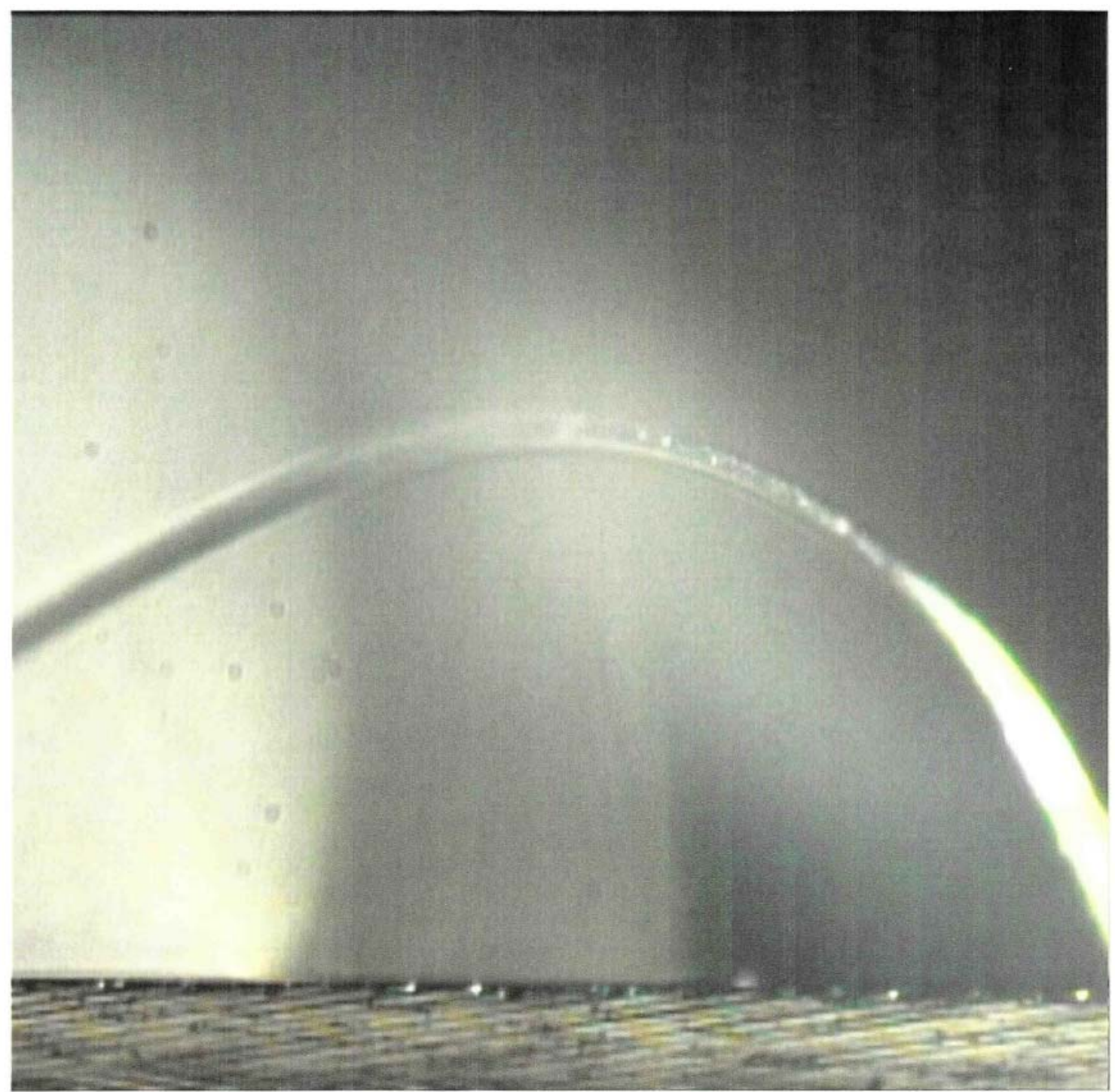

Figure 6 Magnified wire bond wire picture on video display. 


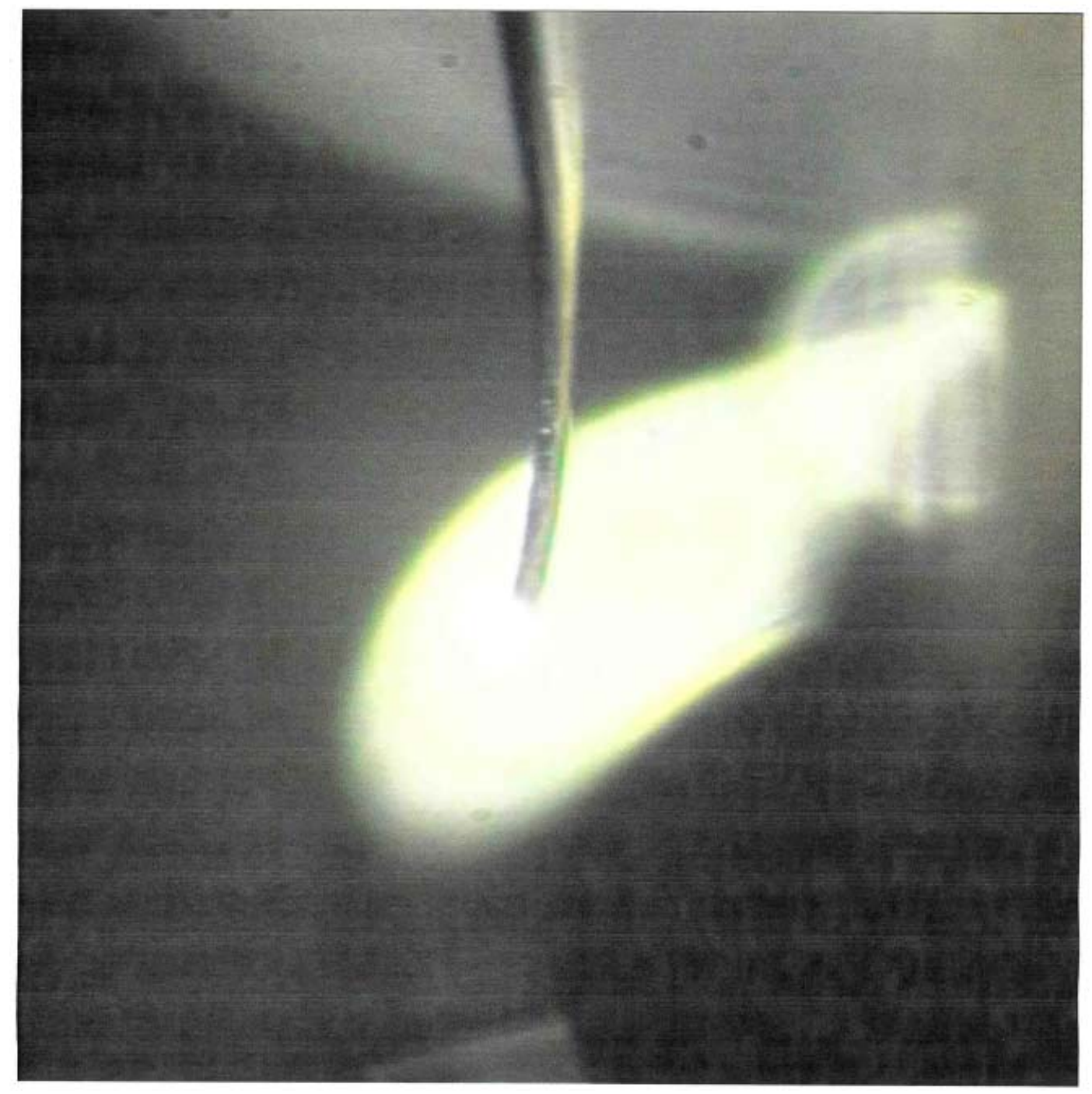

Figure 7 A magnified picture shows one of the broken wire bond wires. 